\title{
PARALLEL SEQUENCE SPREAD SPECTRUM SYSTEM SIMULATION WITH RAPP MODEL
}

\author{
Karthik KrishneGowda ${ }^{1}$, Abdul Rehman Javed ${ }^{2}$, Andreas C. Wolf ${ }^{3}$ and \\ Rolf Kraemer ${ }^{4}$ \\ ${ }^{1}$ Brandenburg University of Technology, Cottbus, Germany \\ Karthik.Krishnegowda@b-tu.de \\ ${ }^{2}$ Heinz Nixdorf Institut, Universität Paderborn, Paderborn Germany \\ arjaved@hni.uni-paderborn. de \\ ${ }^{3}$ Dr. Wolf Wireless GmbH, Teltow, Germany \\ aw@dw-w. com \\ ${ }^{4}$ IHP Microelectronics, Frankfurt (Oder), Germany \\ kraemerdihp-microelectronics.com
}

\begin{abstract}
In Terahertz frequency band there is an abundant of bandwidth available to achieve ultra-highspeed wireless communication to achieve data rates of 100 Gbps. We choose Parallel Sequence Spread Spectrum (PSSS) as an analog friendly modulation and coding scheme that allows for an efficient mixed-signal implementation of a 100 Gbps wireless baseband. Rapp Model is a 'behavioural amplifier model' which characterize the AM/AM conversion of a solid-state high power amplifier. In this paper, a PSSS modulated signal is transmitted through a "Rapp Model", then through an AWGN channel and finally a PSSS demodulator which recovers the signal and performs BER calculations. We have to investigate as on how much non-linearity induced by PA can PSSS modulated system tolerate and still be able to recover the transmitted data at the receiver.
\end{abstract}

\section{KEYWORDS}

PSSS, Rapp Model, 100 Gbps, PA, wireless, Terahertz

\section{INTRODUCTION}

Wireless systems are a big driver of new and challenging research directions. The IEEE P802.15.3d TG3d [1] defines an application for wireless point-to-point (P2P) physical layer operating data rates up to $100 \mathrm{Gbps}$. The emerging wireless P2P applications are data centers, wireless backhaul/fronthaul services, intra-device communication, and close proximity $\mathrm{P} 2 \mathrm{P}$ application [1].

In a decade 2020-2030, projected Wireless LAN (local area network) data rates is set to increase by 100x [2]. As in the Figure 1, the data rate requirement for WLAN in 2025 is about $1 \mathrm{~Tb} / \mathrm{s}$. 
Thus, there is a need to move for the higher end of spectrum where we have more available bandwidth.

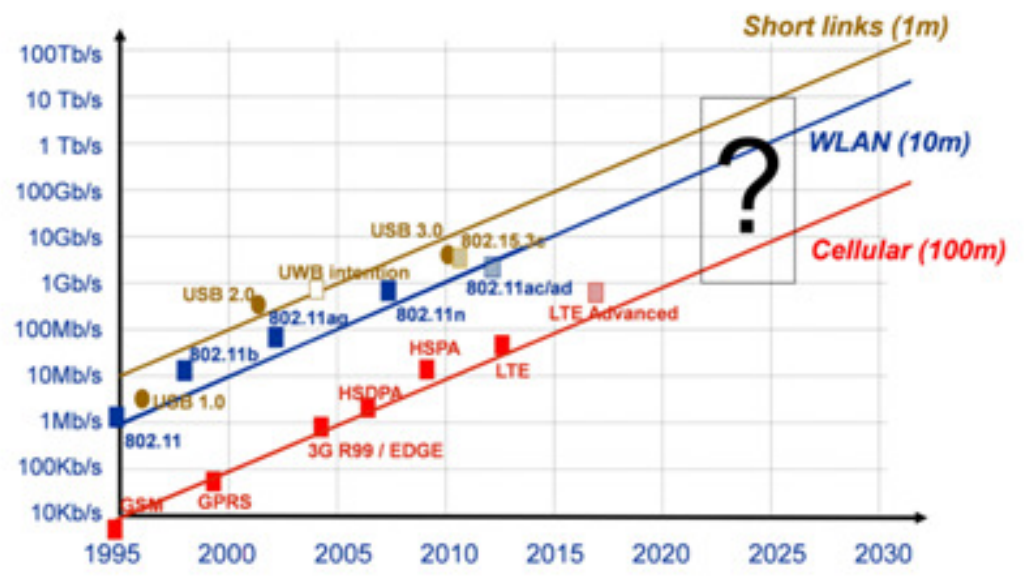

Figure 1 : The Wireless Roadmap: Race for Data Rate [2]

Parallel sequence Spread Spectrum (PSSS) initially published by Wolf [3, 10, 11], has promising features with lower complexity and higher throughput. We employ a novel mixed-mode design where baseband signal processing can be done mostly in analog domain and partly in digital domain, which helps reducing the power consumption [4, 5]. In the $240 \mathrm{GHz}$ frequency band there is about $55 \mathrm{GHz}$ of contiguous bandwidth available, such that with a spectral efficiency of $2-3 \mathrm{bit} / \mathrm{s} / \mathrm{Hz}$ we can achieve $>100 \mathrm{~Gb} / \mathrm{s}$.

RF power amplifiers (PA) are important elements in transmitter used in wireless communication. PA saturates beyond a threshold of power which results in clipping of signals and thus causing non-linear distortion. A general method to compensate for clipping effects is to reduce the input drive level so that operating point falls in the linear region of the PA [6, 7]. However, by operating PA with high back-off reduces the energy efficiency of the transmitter. Thus, there is a tradeoff between PA's efficiency and linearity. In this paper, we model the power amplifier characteristics using Rapp Model [8].

\section{PSSS BASEBAND}

Our research in mixed-signal processing for ultra-broadband transceivers is based on the assumption that a partitioning of digital-/ analog- signal processing in the baseband with more focus on analog processing has the potential to outperform purely digital baseband processors in terms of power dissipation, complexity, and cost. We chose PSSS as an analog-friendly modulation and coding scheme. Figure 2 shows the basic concept of a PSSS transmitter and receiver and allows explaining the advantages of a mixed-signal PSSS baseband implementation. 


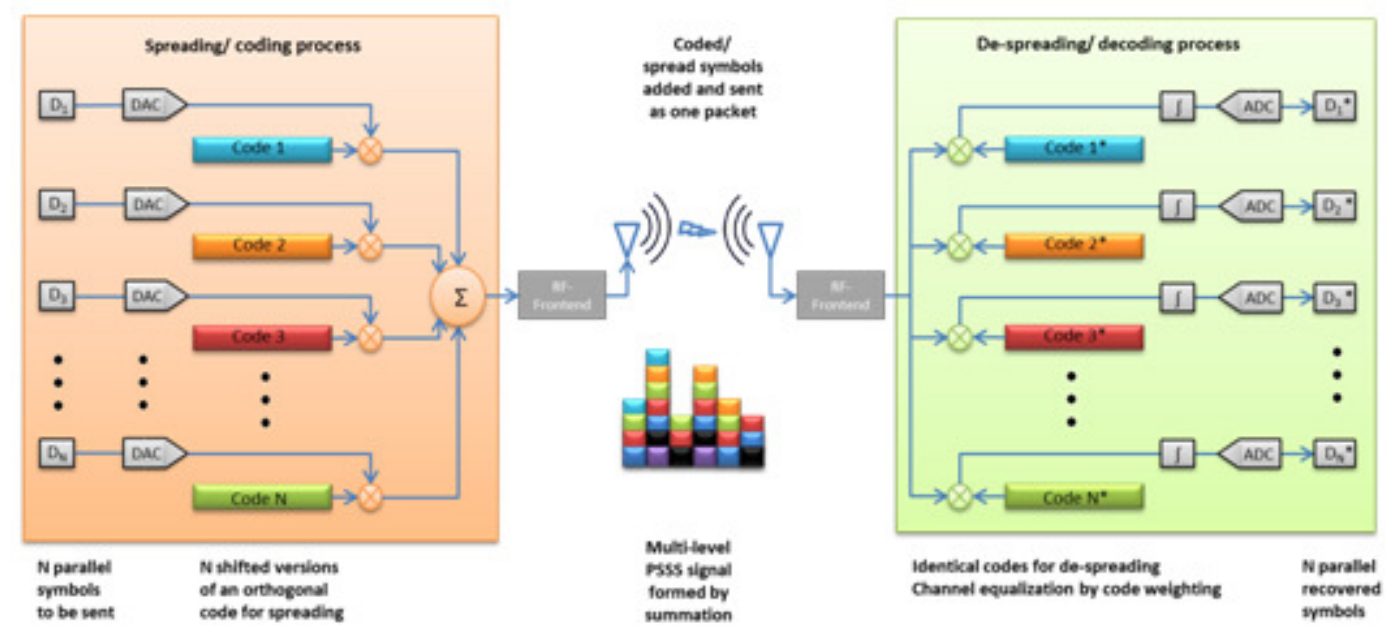

Figure 2: Mixed-Signal PSSS transceiver concept [10]

In the PSSS transmitter N parallel symbols D1 to DN are encoded with $\mathrm{N}$ orthogonal codes and summed up to a single quantized PAM-signal. These signals are up-converted to RF transmit-ted over the radio channel, and down-converted to baseband. The receiver cross-correlates the PSSS baseband signal with the N PSSS codes and recovers the symbols D1 to DN. The com-plete spreading-/coding-process in the transmitter and despreading-/decoding-process in the receiver can be implemented very efficiently in analog domain using fast analog circuitry, such as analog multipliers, adders, and integrators. In the mixed-signal PSSS baseband from Figure $2 \mathrm{~N}$ parallel DACs and ADCs are utilized operating at a sampling frequency equal to symbol rate. On the contrary, in a fully digital PSSS transceiver the DAC and the ADC would have to process the encoded PSSS signal, i.e. to operate with a sampling frequency of at least $2 x$ the PSSS signal bandwidth.

A further advantage of a mixed-signal PSSS transceiver is that adaptive equalization of the received signal can be implemented in the despreading/decoding process [10] as a crosscorrelation-based equalization. Decoding the received signal with a replica of a distorted PSSS code we obtain a perfect correlation result, effectively compensating the non-ideal channel impulse response.

\section{RAPP MODEL}

A widely accepted solid state power amplifier model encompassing the amplitude clipping (i.e., AM-AM distortion) is the Rapp Model [8]. Rapp Model is designed according to the Equation (1) and it produces a smooth transition for the envelope characteristic as the input amplitude approaches saturation. Amplitude distortion (AM/AM) and Phase distortion (AM/PM) conversion are given according to Equation (1)

$$
A_{\text {out }}=\frac{\boldsymbol{A}_{\text {in }}}{\left[1+\left[\frac{\left|\boldsymbol{A}_{\text {in }}\right|^{2 p}}{\boldsymbol{A}_{\text {sat }}}\right]\right]^{1 / 2 p}}, \theta=0
$$


wherein $A_{\text {sat }}$ is the saturation output amplitude, $A_{\text {in }}$ is the input amplitude, $A_{\text {out }}$ is the output amplitude, and $\mathrm{p}$ controls the smoothness of the transition from a linear region to a saturation region.

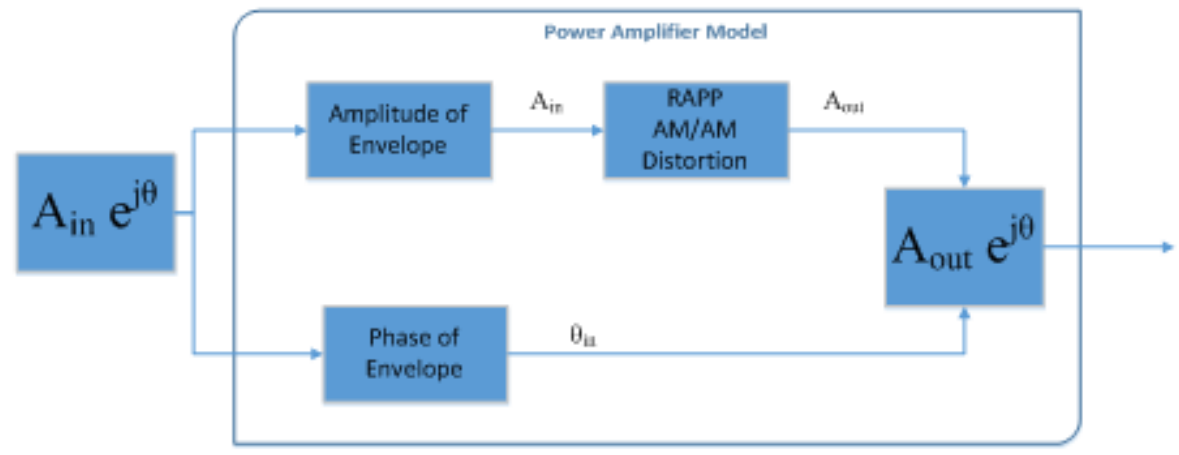

Figure 3: Rapp Model

Figure 3 shows the Rapp model of the PA recommended in IEEE 802.11 [9], envelope of the input signal is measured using "Amplitude of Envelope" which is then passed on to "RAPP AM/AM Distortion" wherein input amplitude is clipped off according when it reaches saturation point and the phase of the input signal is unaltered.

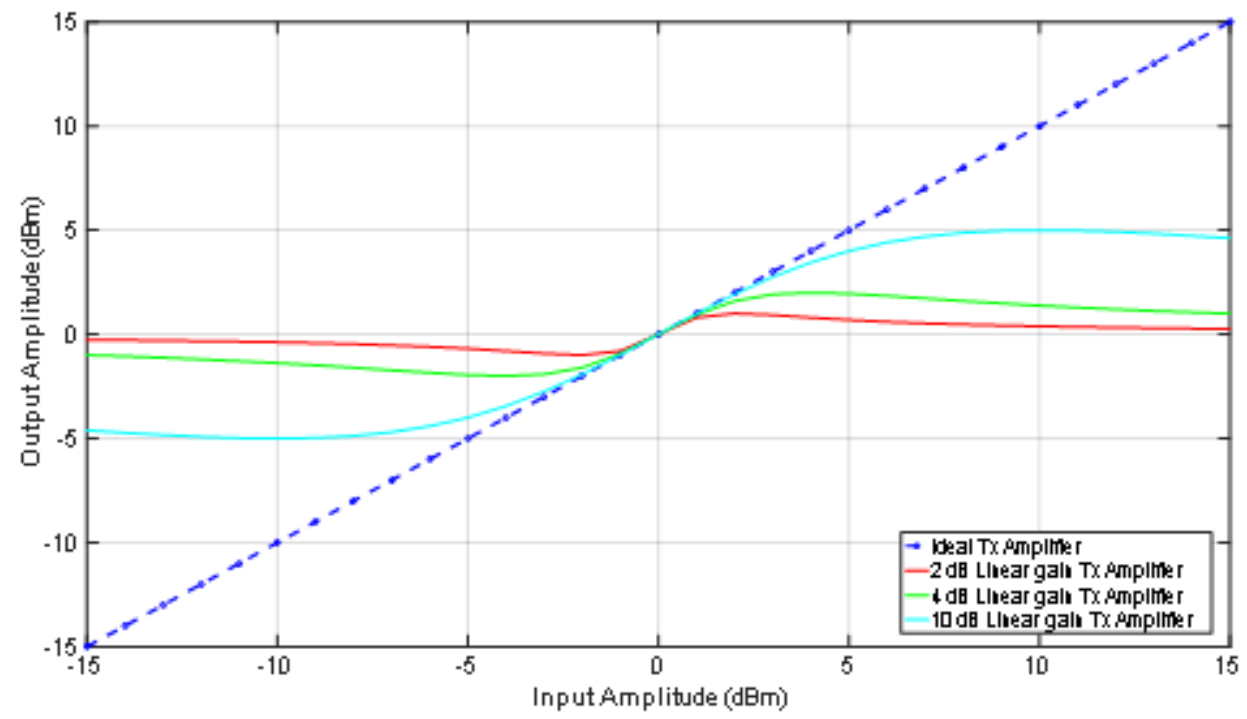

Figure 4: AM/AM response of Power Amplifier

Figure 4 shows the AM/AM response of the Rapp model as described in equation (1). It is clear from the graph, as we increase the linear gain of the transmit amplifier will leads to reduction in clipping of the input signal. For e.g., a $10 \mathrm{~dB}$ linear gain TX amplifier does not clip off the input amplitudes between $-5 \mathrm{dBm}$ and $+5 \mathrm{dBm}$ and it clips off of any inputs amplitude which does not fall in these range. 


\section{Simulation SETUP}

The PSSS system level model which includes the PSSS transmitter, PSSS receiver, synchronisation, and channel equalizations is described in our paper [4]. Figure 5 shows simulation model used in the experiments. The data bits are modulated by PSSS modulator and followed by a "Rapp Model" which introduces clipping in amplitude caused due to non-linear PA. Then, data is passed through an AWGN channel and finally demodulated by PSSS demodulator which evaluates for BER.

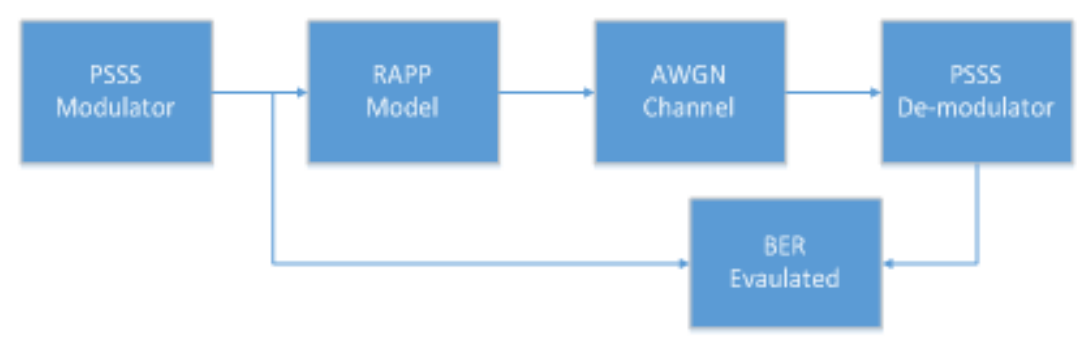

Figure 5: System Simulation Model

The signal processing of the transmitter-model (as in Figure 2) generates parallel streams of data that are encoded using m-sequences of length of 15 (PN sequences). The parallel streams were summed up subsequently to obtain a 'multi-level PSSS signal'. In Figure 6, the x-axis represents the different discrete amplitude values at the output of "PSSS Modulator" (as in Figure 5) and Yaxis shows of the number of occurrence of these amplitudes for all input combinations of data bits. From the distribution it becomes obvious that most information (90\%) in the encoded signal is present in the lower amplitudes of 'multi-level PSSS Signal' and the encoded information content goes down (10\%) as we move to higher amplitudes 'multi-level PSSS Signal'. We have to investigate how much clipping we can perform (i.e. how much non-linearity induced by PA can PSSS modulated system can tolerate) on this 'multi-level PSSS Signal' and still be able to recover the transmitted data at the receiver.

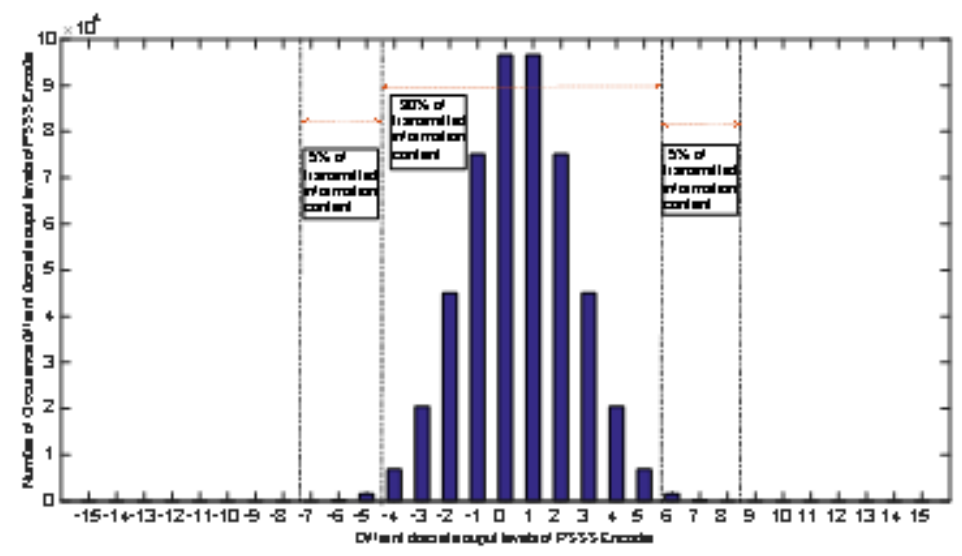

Figure 6: Bar graph depicting different levels amplitudes and their occurrence frequency 


\section{RESULTS}

PSSS system was modulated with a chip rate of $20 \mathrm{Gcps}$ and spectral efficiency of $1 \mathrm{bit} / \mathrm{s} / \mathrm{Hz}$. As shown in the Figure 7, to achieve BER of $1 \mathrm{e}^{\wedge}-4$ we need SNR $14.5 \mathrm{~dB}$ with PA having linear gain of $2 \mathrm{~dB}$ (PA characteristics as shown in Figure 4) whereas the required SNR drops down to $12 \mathrm{~dB}$ with PA having linear gain of $10 \mathrm{~dB}$. Thus, with less SNR we can achieve same BER $\left(1 \mathrm{e}^{\wedge}-4\right)$ using high linear gain transmit amplifier.

One more important point is, even if we have very poor PA with linear gain of $2 \mathrm{~dB}$ (PA characteristics as shown in Figure 4), PSSS demodulator is still able to recover the signal at the cost of increased SNR. For e.g., with $2 \mathrm{~dB}$ linear gain of PA, we could achieve BER of $1 \mathrm{e}^{\wedge}-4$ while SNR increases to $14.5 \mathrm{~dB}$. Thus, PSSS modulation/demodulation can tolerate the clipping caused by the PA.

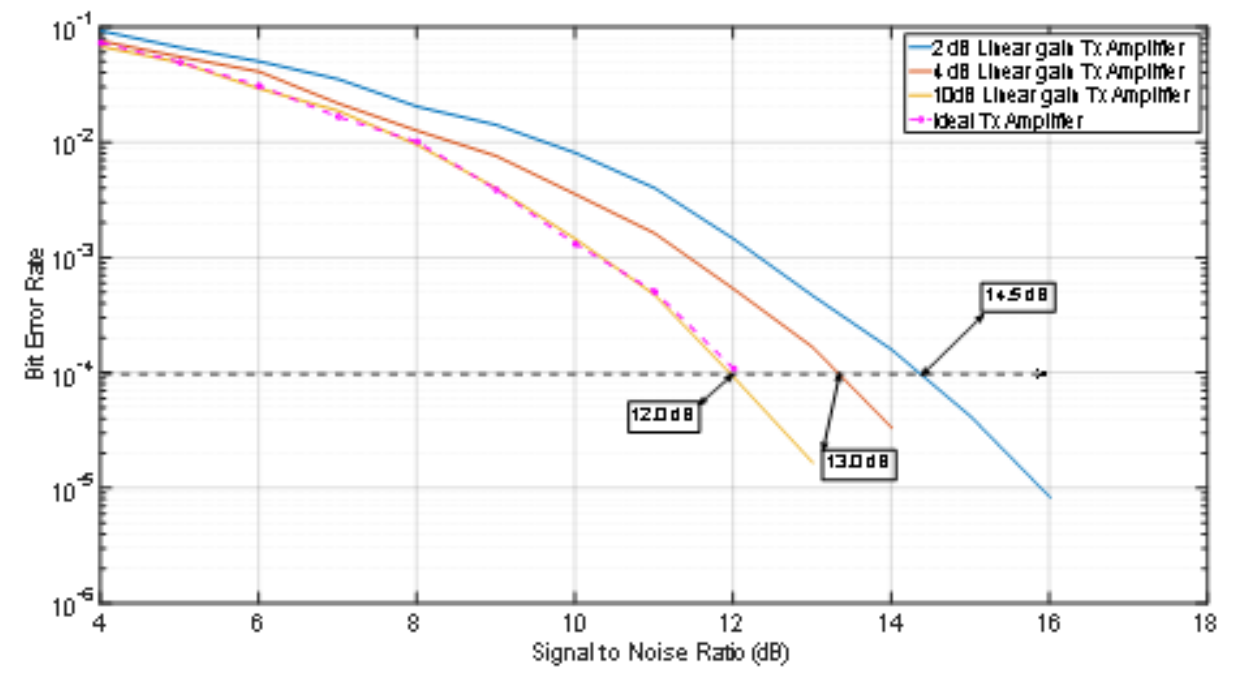

Figure 7: BER vs SNR for PSSS modulated signal with chip rate of $20 \mathrm{Gcps}$ with different linear gains of the Transmit Power Amplifier

\section{CONCLUSIONS}

We have shown that PSSS modulated baseband system can accept the non-linear impairments like clipping caused by PA and be still able to recover the transmitted signal. This shows the high robustness PSSS modulation and its inherent ability to correct these errors induced by performing adaptive channel equalisation $[4,5]$ at the receiver. In our simulations, we were able to transmit the signal with a PA having linear gain of $2 \mathrm{~dB}$ and achieve BER $1 \mathrm{e}^{\wedge}-4$. PSSS modulation is best suited to achieve high data rates due its inherent error tolerating properties.

\section{ACKNOWLEDGEMENTS}

This paper is related to Real100G.com project and cooperates with other proposed projects of the DFG Special Priority Program 1655 (SPP1655) on "Wireless 100Gbps and beyond", e.g. the End2End100 and Real100G.RF 


\section{REFERENCES}

[1] IEEE P802.15.3d TG3d (100G), "IEEE 802.15 Documents." [Online]. Available: https://mentor.ieee.org/802.15/documents

[2] Fettweis, Gerhard P. "The tactile internet: applications and challenges." IEEE Vehicular Technology Magazine 9.1 (2014): 64-70.

[3] Wolf, A.: 'PSSS Patents EP04701288.5-1515/1584151, DE 102004033 581, US 20060256850'.

[4] KrishneGowda, K.; Kraemer, R.; Wolf, A.; Scheytt, J.C.; Kallfass, I., "Wireless 100 Gb/s: PHY layer overview and challenges in the $\mathrm{THz}$ frequency band," Wireless and Microwave Technology Conference (WAMICON), 2014 IEEE 15th Annual, vol., no., pp.1,4, 6-6 June 2014 doi: 10.1109/WAMICON.2014.6857743

[5] Javed, Abdul Rehman; Scheytt, J.Christoph; KrishneGowda, Karthik; Kraemer, Rolf, "System design considerations for a PSSS transceiver for 100Gbps wireless communication with emphasis on mixed signal implementation," Wireless and Microwave Technology Conference (WAMICON), 2015 IEEE 16th Annual, vol., no., pp.1,4, 13-15 April 2015

[6] M. Shabany and P. G. Gulak, "Efficient Compensation of the Nonlinearity of Solid-State Power Amplifiers Using Adaptive Sequential Monte Carlo Methods", IEEE Trans. Circuits Syst., I: Reg. Papers, vol. 55, no. 10, pp. 3270-3283, Nov. 2008

[7] Z. Hasan, H. Boostanimehr, and V. K. Bhargava, "Green cellular networks:A survey, some research issues and challenges," IEEE Commun.Surveys \& Tuts., vol. 13, no. 4, pp. 524-540, Fourth Quarter, 2011

[8] C. Rapp, "Effects of HPA-nonlinearity on a 4-DPSK/OFDM signal for a digital sound broadcasting system," Proc. 2nd European Conf. Satellite Communications, pp. 179-184, Oct. 1991.

[9] Webster, Mark. "Suggested PA Model For 802.11 Hrb". N.p., 2000. [Online] Accessed 25 Aug. 2016 http://goo.gl/ckrDL0

[10] A. C. Wolf and C. Scheytt, "15 Gbps communication over an USB3.0 cable and even more," in Systems, Signals and Devices (SSD), 2012 9th International Multi-Conference on, 2012, pp. 1-3.

[11] A. Wolf, R. Kraemer, and J. C. Scheytt, "Ultra high speed wireless communication with low complexity transceiver," in Signals, Systems, and Electronics (ISSSE), 2012 International Symposium on, 2012, pp. 1-6. 